\title{
Albrecht von Graefe (1828-1870) und die Ophthalmopathologie
}

\section{Albrecht von Graefe (1828-1870) and Ophthalmic Pathology}

„Fassen wir die Hauptvortheile, welche uns die durchsichtige Beschaffenheit des Auges verschafft, zusammen, so liegen sie in der innigen Verschmelzung klinischer und pathologisch-anatomischer Beobachtung, einer Verschmelzung, welche gerade die neuere Medicin mit allen Kräften, aber mit sehr ungleichem Erfolge in den verschiedenen Disciplinen anstrebt“. (Albrecht von Graefe, 1865 [1])

Albrecht von Graefe ( Abb. 1) gilt als Begründer der modernen Augenheilkunde und immer noch als berühmtester Augenarzt weltweit. Dementsprechend sind zahlreiche Biografien über ihn verfasst worden [2-4]. Die erste Lebensbeschreibung erfolgte 1877 durch seinen Schüler und Mitarbeiter Eduard Michaelis (18241891) [5], die letzte 2013 im Rahmen der Graefe-Donders-Briefedition [6]. Albrecht von Graefe begründete 1854 das „Archiv für Ophthalmologie“ (seit 1871 „Albrecht von Graefes Archiv für Ophthalmologie, heute Graefes Archive for Clinical and Experimental Ophthalmology) und 1857 die Deutsche Ophthalmologische Gesellschaft (DOG). Darüber hinaus inspirierte er maßgeblich die „Geburt“ der „Klinischen Monatsblätter“ 1863 [7]. In Berlin erinnern heute die „Graefe-Straße“ in Kreuzberg und vor allem das monumentale Graefe-Denkmal ( $\mathbf{A b}$ b. 2) an den bedeutenden Fachkollegen. Sein Grab auf dem Friedhof II der Jerusalems- und Neuen Kirchengemeinde in Kreuzberg wird vom Land Berlin als Ehrengrab gepflegt. Die DOG vergibt alle 2 Jahre den GraefePreis und alle 10 Jahre die Graefe-Medaille.

Albrecht von Graefe beschäftigte sich immer mit der gesamten Augenheilkunde, jedoch standen für ihn meist bestimmte Themen im Vordergrund. Sein Schüler Julius Hirschberg (1843-1925) differenzierte 3 Schaffensperioden [8]. In der 1 . Periode bis 1857 dominierten Forschungen zu Erkrankungen der Bindehaut, zur Sinnesphysiologie und zum Strabismus, mit denen er sich 1852 habilitierte („Über die Wirkung der Augenmuskeln“). Albrecht von Graefe war einer der Allerersten, der sich des 1851 von Hermann von Helmholtz (1821-1894) vorgestellten Augenspiegels bediente, und so gelangen ihm z. B. die Erstbeschreibung der glaukomatösen Papillenexkavation 1855 [9] oder des Zentralarterienverschlusses 1859 [10]. Daher hält die Graefe-Statue im Denkmal auch einen Augenspiegel in der Hand (ه Abb. 2). In der 2. Periode bis etwa 1863 standen die Forschungen zum Glaukom im Vordergrund. Die Einführung der peripheren Iridektomie in die Glaukombehandlung 1857 [11] gilt als seine größte ophthalmologische Leistung überhaupt [12]. In der 3. Periode waren für Graefe Fragen der Kataraktextraktion vorrangig. Seine letzte, große Arbeit befasste sich indes noch einmal mit dem Glaukom [13]. Die Ophthalmopathologie wird im Allgemeinen nicht unmittelbar mit seinem Wirken verbunden, hatte für Albrecht von Graefe aber durchaus große Bedeutung, wie im Folgenden beleuchtet werden soll. Bereits im Vorwort zum 1. Band seines Archivs schrieb Albrecht von Graefe, die Ophthalmomorphologie hervorhebend:

„... die sich häufenden anatomischen und pathologisch-anatomischen Untersuchun-

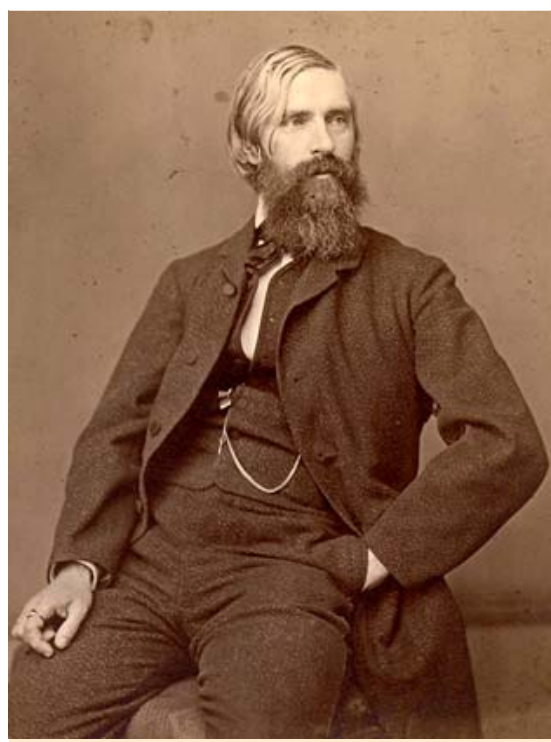

Abb. 1 Albrecht von Graefe, um 1865 (Bild aus dem Archiv des Autors).

gen, die zahlreichen physiologischen Arbeiten, vor allen Dingen aber die Erfindung des Ophthalmoskops, welche den Namen Helmholtz mit dem Lorbeer der Unsterblichkeit schmückt, geben dem Fache einen literarischen Aufschwung, dem selbst der Ophthalmologe nur mit Anstrengung aller Kräfte $\mathrm{zu}$ folgen im Stande ist“ [14].

Zu diesem Zeitpunkt lagen die Besetzung des weltweit ersten Lehrstuhls für Pathologie an der Universität Straßburg mit Jean-Frédéric Lobstein (1777-1835) gerade einmal 35 Jahre, die Prägung des Begriffs „Zelle“ durch Jakob Schleiden (1804-1881) und die Einführung des Mikroskops in die morphologische Diagnostik etwa 25 Jahre sowie die Klassifikation der Gewebe durch Jakob Henle

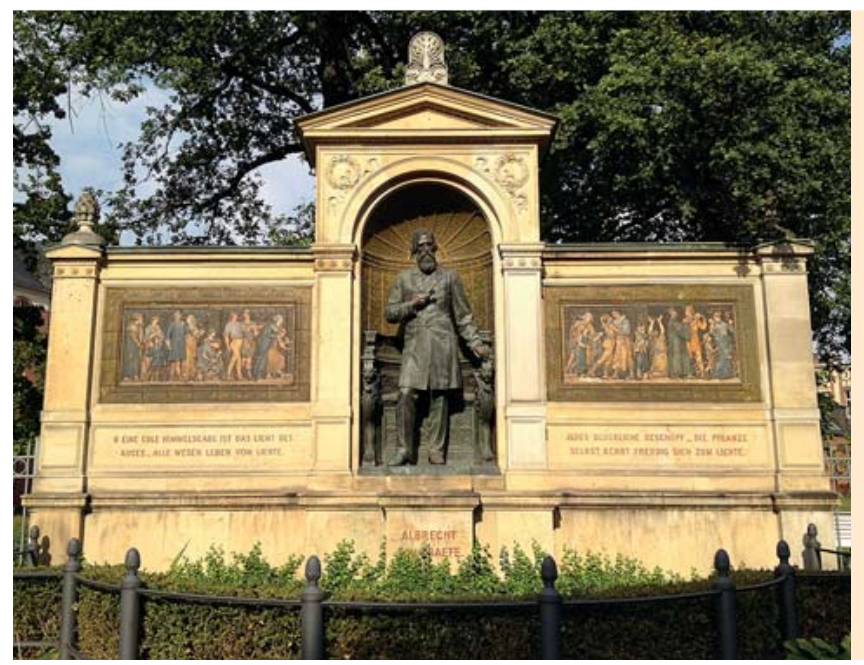

Abb. 2 Graefe-Denkmal am Rand des Charité-Geländes, Ecke Schumann- und Luisenstraße. Das Denkmal wurde am 22. Mai 1882 feierlich eingeweiht [6] Dargestellt ist der Kliniker Albrecht von Graefe. Seine grundlagenwissenschaftlichen Arbeiten auf dem Gebiete der Physiologie des Sehens und der Ophthalmopathologie blieben vor dem Hintergrund der damaligen Zeit aus verständlichen Gründen - unberücksichtigt. 


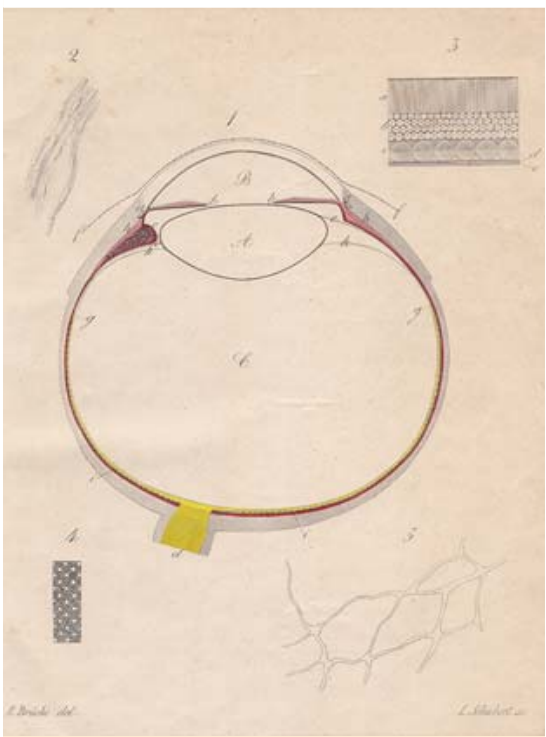

Abb. 3 Ernst Wilhelm Brücke: Anatomie des Auges, 1847. Brücke entdeckte 1846 die äußeren, meridionalen Fasern des Ziliarmuskels, die später nach ihm benannt wurden („Brücke-Muskel“). Die Differenzierung der verschiedenen Portionen des Ziliarmuskels ist, da die inneren zirkulären Fasern (,Müller-Muskel“) noch unbekannt waren, in der Bulbuszeichnung unterblieben (vgl. auch $\bigcirc$ Abb. 4). Im Randbereich erste „rudimentäre" histologische Abbildungen, so (rechts oben) der Netzhaut mit den Fotorezeptoren oben und der Membrana limitans interna unten; aus [16].

(1809-1885) gerade einmal 15 Jahre zurück [15]. Rudolf Virchows (1821-1902) „20 Lehrgänge der Cellularpathologie“, brauchbare Mikrotome und die Entwicklung guter Fixier-, Einbett- und Färbemethoden als Voraussetzung für eine verlässliche Histologie sollten erst noch (bis etwa 1900) kommen. Woher also Graefes Faible für die Ophthalmopathologie? Sehr wesentlich dafür dürfte gewesen sein, dass er während seines Studiums an der Universität Berlin 1843-1848 vom berühmten Physiologen, Anatomen und Wegbereiter der modernen Medizin, Johannes Müller (1801-1858) und vom jungen Physiologen Ernst Wilhelm Brücke (1819-1892), der 1847 eine der ersten zusammenfassenden Darstellungen der Anatomie des Auges herausgab [16] ( $\odot$ Abb. 3), beeinflusst worden war [6]. In ganz besonderem Maße soll der Student Graefe vom jungen Dozenten Virchow gefesselt worden sein $[2,8]$. Beide, Graefe und Virchow, der im Übrigen 1843 mit einer Arbeit über die Kornea promoviert wurde, verband eine lebenslange Freundschaft. Dieses, obwohl sich Virchow im Lehrkörper der Universität Berlin gegen die von Albrecht von Graefe für notwen-

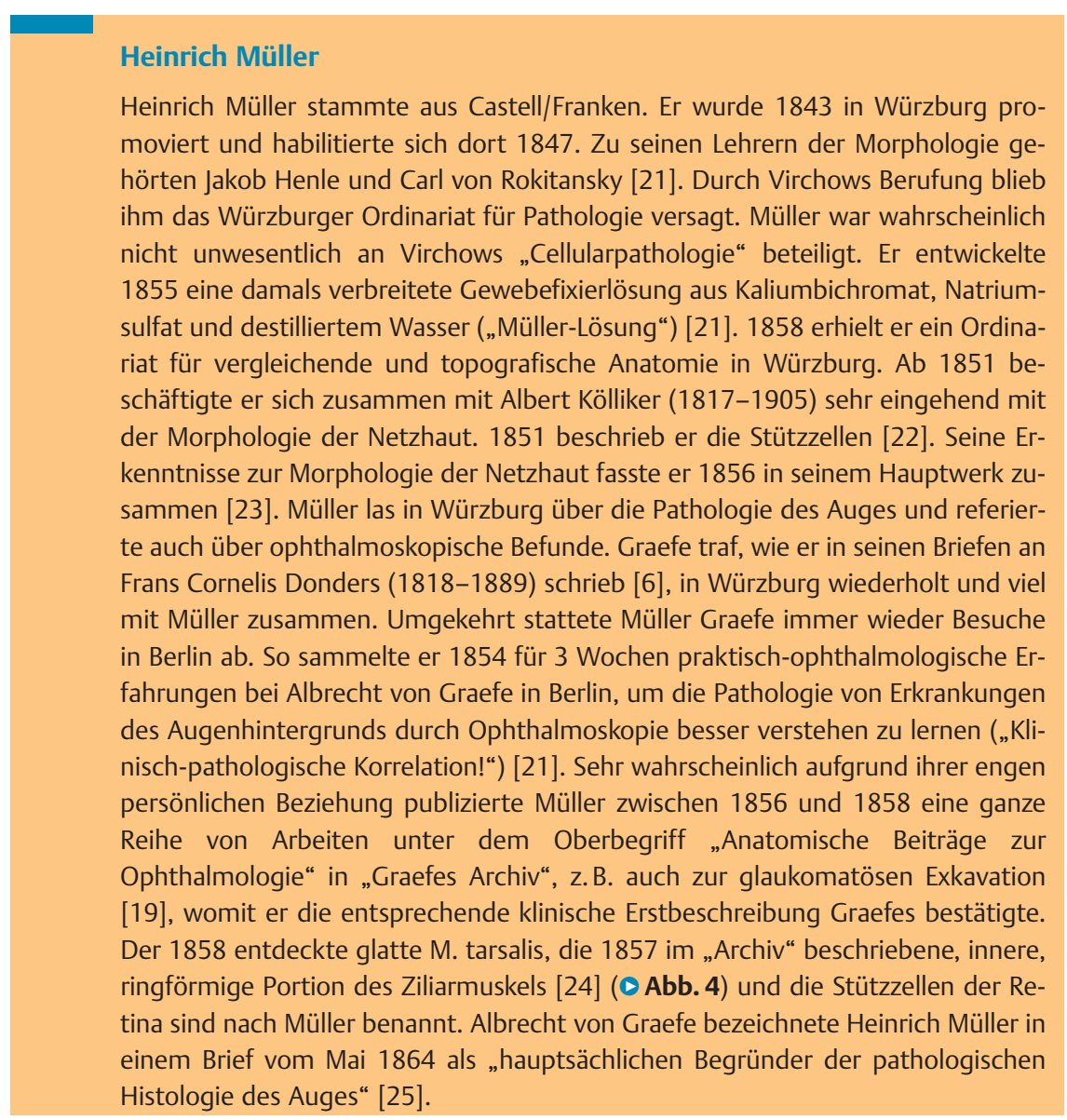

dig erachtete Verselbständigung der Augenheilkunde aussprach, worüber sich letzterer in einem Brief an seinen Schüler und Freund Julius Jacobson (1828-1889) von 1868 recht bitter beschwerte [17]. Virchow ging aufgrund seiner aktiven Beteiligung an der Märzrevolution von 1848 im Jahre 1849 ins „Würzburger Exil“, in dem er seine "Cellularpathologie“ schrieb. Noch 1858, als Albrecht von Graefe als Augenarzt zu Weltruhm gekommen war, soll er die Vorlesungen des großen Pathologen besucht haben [8]. Während seiner „Wanderjahre“ hörte Albrecht von Graefe in Wien 1850 neben der Augenheilkunde vor allem Pathologie bei Carl von Rokitansky (1804-1878), der besonderen Wert auf die Verzahnung von Klinik und Morphologie legte [5]. Schließlich war auch der sehr enge Londoner Freund William Bowman (18161892), bevor er sich der Augenheilkunde zuwandte, Anatom und Mikroskopiker gewesen, was den „morphologischen Gedankenaustausch" ebenfalls angeregt haben dürfte.

Anfang 1852 eröffnete Albrecht von Graefe seine private Augenklinik in der Berliner Karlstraße (heute Reinhardt- straße). Ob in dieser Klinik selbst Ophthalmopathologie betrieben wurde, ist unklar. Graefe schickte auf jeden Fall interessante, exzidierte Gewebe oder Augen (auch) nach Würzburg zu Virchow und Heinrich Müller (1820-1864) $[6,18,19]$. Befördert wurden diese Kontakte sicher durch die in Paris entstandene, enge Freundschaft mit dem in Würzburg praktizierenden Robert Ritter von Welz (1814-1878), der 1866 erster Ordinarius für Augenheilkunde in Würzburg wurde, und durch seine Beziehung mit der Würzburgerin Katharina Vogel (1827-1915), mit der zusammen er die (voreheliche) Tochter Maria Antonie Mathilde (18541909) hatte [20]. Wiederholt machte Graefe während seiner Sommerurlaube in Würzburg Station und operierte zusammen mit Welz [20]. Nachdem Rudolf Virchow 1856 nach Berlin zurückgekehrt war und das dort neu geschaffene Ordinariat für Pathologie übernommen hatte, wurden Graefes Gewebeproben wieder vor Ort in Berlin aufgearbeitet. Im „Lehrkursus in der Augenklinik des Doktor von Graefe“ 1855 wurde „Anatomie und Histologie des Auges“ gelesen und „Übungen in der mikroskopischen Unter- 
suchung des Auges“ durchgeführt. Dieses allerdings durch den Graefe-Schüler Richard Liebreich (1830-1917) [2]. Graefe selbst kümmerte sich um die „Klinik der Augenkrankheiten“ und die „OperationsÜbungen“, aber allein die Aufnahme der Morphologie in den Unterrichtsplan unterstreicht, wie sehr diese Graefe am Herzen lag.

Sieht man die zu Lebzeiten von Graefe herausgegebenen Bände des „Archivs“ und der „Monatsblätter“ (also 1854-1870 bzw. 1863-1870) durch, so stellt man zunächst erwartungsgemäß fest, dass er in seinem „Archiv“ deutlich mehr Arbeiten veröffentlichte. Seine Publikationen hatten fast ausnahmslos einen klinischen oder operationstechnischen Hintergrund. Umfangreichere Arbeiten Graefes überwiegend oder gar rein ophthalmopathologischen Inhalts sucht man in beiden Journalen vergeblich. Wohl aber haben seine Mitarbeiter Richard Liebreich, Carl Schweigger (1830-1905) [26], Theodor Leber (1840-1917) [27] und Julius Hirschberg [28] zahlreiche histologische Arbeiten publiziert, an denen Graefe mit sehr großer Wahrscheinlichkeit beteiligt war, ohne aber, weil seinerzeit nur „Mono-Autorenschaft" üblich war, im Titel zu erscheinen. Bei den unter seinem Namen publizierten Beiträgen wurden die morphologischen Untersuchungen wohl meist von den Mitarbeitern, die er dann namentlich zu erwähnen pflegte, bewerkstelligt $[29,30]$. In einem Vorwort $\mathrm{zu}$ einer morphologischen Abhandlung Schweiggers schrieb Graefe einmal „Obwohl die Arbeit gemeinschaftlich von Dr. Schweigger und mir unternommen ist, so gehören die anatomischen Untersuchungen lediglich Dr. Schweigger an, was ich vorweg zu erklären mich für verpflichtet halte“ [31]. Wir können also davon ausgehen, dass Albrecht von Graefe bei allem Interesse an der Morphologie vor allem seine Mitarbeiter „ophthalmopathologisch machen ließ". Gelegentlich wurde die Morphologie in den Arbeiten aus der Graefeschen Klinik bildlich auf Tafeln dargestellt, wobei der Schwerpunkt auf der Makroskopie lag [32] (O Abb.5). Details konnten naturgemäß noch nicht so aufgelöst werden, wie das heute der Fall ist. Vor allem Heinrich Müller gelang es aber zu Graefes Zeiten schon sehr gut, mikroskopische Befunde am Auge detailliert zu beschreiben [33] ( $\triangle$ Abb. $\mathbf{6}$ ).

Es wäre übertrieben, Albrecht von Graefe als Ophthalmopathologen zu rezipieren. Kein Zweifel kann aber daran bestehen, dass er ophthalmomorphologischen Un-

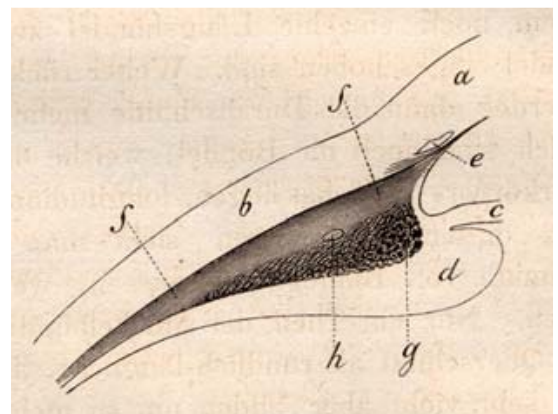

Abb. 4 Abbildung des (mit "g“ gekennzeichneten) Müller-Muskels aus Müllers Publikation von 1857 [24]. Die Darstellung ist, wie damals weithin üblich, "semi-mikroskopisch". Zwar sind die verschiedenen Muskelportionen gut differenzierbar, Details wie z. B. die Zweischichtigkeit des Ziliarkörperepithels sind aber nicht wiedergegeben.

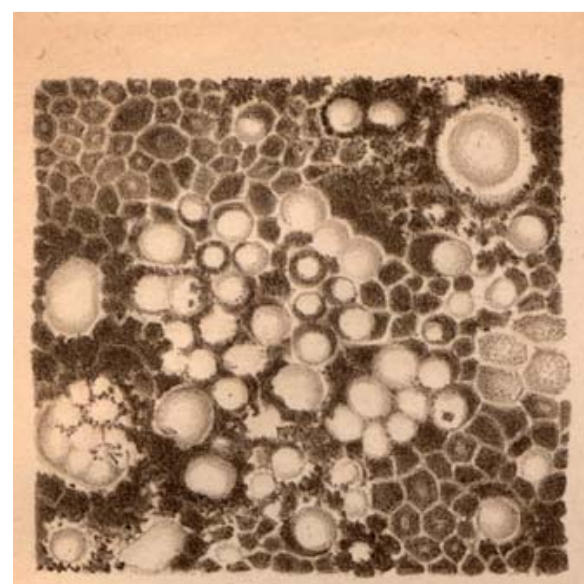

Fig. I.

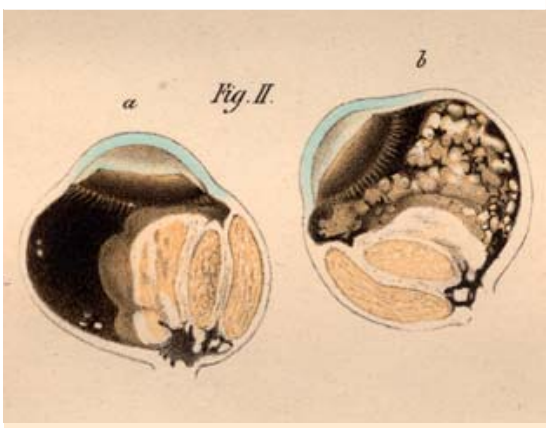

Abb. 5 Makroskopische Darstellung eines tumorösen Prozesses in einem Schweineauge, der von Graefe als „Chorioiditis tuberculosa“ gedeutet wurde. Die Histologie wurde (grob) beschrieben, aber nicht abgebildet. Es handelte sich um einen Zufallsbefund. Das Auge sollte, wie Graefe schrieb, „zu Operationsübungen benutzt werden“. Das wiederum belegt, dass Albrecht von Graefe schon im 19. Jahrhundert „Wetlabs“ durchführte. Aus [32].

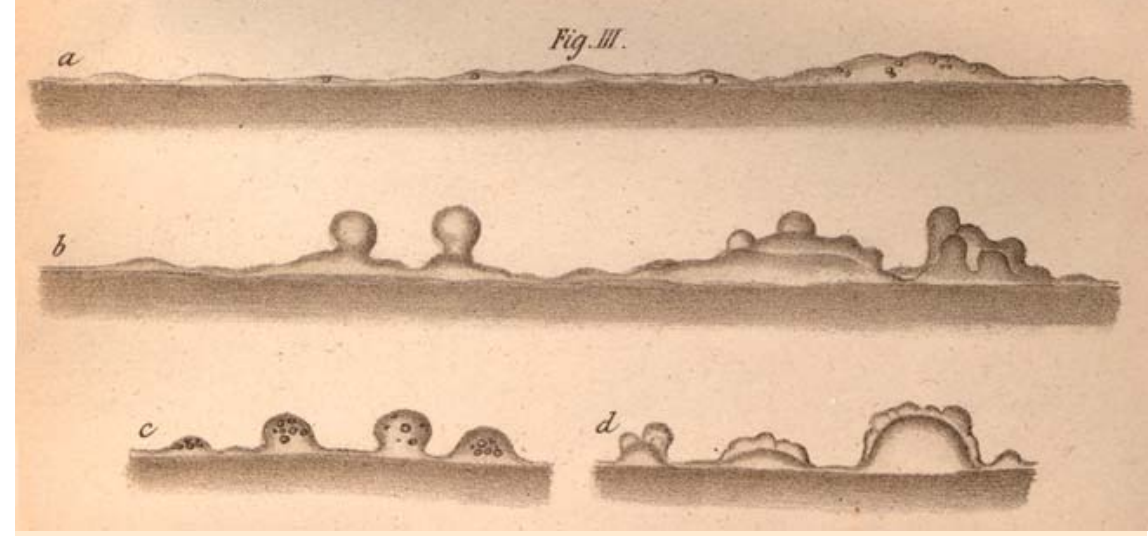

Abb. 6 Detailgetreue Darstellung der senilen Altersveränderungen des retinalen Pigmentepithels (oben) und der Bruch-Membran („Glaslamelle“) mit typischen, von Müller als „Kalkkörner“ bezeichneten Drusen (unten). Aus [33].

tersuchungen einen hohen Stellenwert beimaß, und er und seine Mitarbeiter stets nach der klinisch-pathologischen Korrelation suchten, wie das in den Titeln
„Histologisch-ophthalmoscopische Notizen“ [34] und „Beiträge zur anatomischen Klinik der Augenkrankheiten“ [31] sehr schön zum Ausdruck kam. 
Nach Albrecht von Graefe brachen vor allem Otto Becker (1828-1890), Julius Michel (1843-1911), und Theodor Leber - die beiden letzten Schüler von Graefes - der Ophthalmopathologie im letzten Viertel des 19. Jahrhunderts im Deutschen Reich Bahn [35]. Diese entwickelte sich zu einer ganz wichtigen, von Augenärzten betriebenen Subdisziplin. So blieb es bis 2000. Seit 1-2 Dekaden droht die Ophthalmopathologie vor allem aus ökonomischen Gründen in der (allgemeinen) Pathologie auf- und damit als „Spezialfach" unterzugehen [15,35]. In seiner wegweisenden Rede von 1865 stellte Albrecht von Graefe sehr prägnant heraus, dass sich Augenheilkunde und Medizin wechselseitig befruchten [1]. Gleiches gilt für die Ophthalmopathologie und die Augenheilkunde 150 Jahre später noch immer. Und so befindet sich der heutige, moderne Ophthalmopathologe in allerbester Graefe-Tradition.

\section{Acknowledgements \\ $\nabla$}

Herrn Prof. Dr. G.O.H. Naumann, emeritierter Ordinarius für Augenheilkunde in Erlangen, Ehrenschriftleiter der „Monatsblätter“ und unermüdlicher Protagonist der Ophthalmopathologie in Deutschland, zum 80. Geburtstag mit den besten Wünschen gewidmet.

\section{Interessenkonflikt: Nein.}

\section{J. M. Rohrbach}

Department für Augenheilkunde, Forschungsbereich Geschichte der Augenheilkunde/Ophthalmopathologisches Labor, Eberhard-Karls-Universität Tübingen

\section{Literatur}

1 von Graefe A. Rede über die Bedeutung ophthalmologischer Studien für die Medizin zur Feier des ein und siebenzigsten Stiftungstages des medizinisch-chirurgischen Friedrich-Wilhelms-Instituts am 2. August 1865. Berlin: Hermann Peters; 1865

2 Hirschberg J. Albrecht von Graefe. In: Ziehen J, Hrsg. „Männer der Wissenschaft“, Heft 7. Leipzig: Wilhelm Weicher; 1906

3 Heynold von Graefe B. Albrecht von Graefe. Mensch und Umwelt. München: Karl Thiemig, 1969 (Nachdruck 1990 mit ergänzender „Graefologie“; Berlin: W. Stapp)

4 Münchow W. Albrecht von Graefe. Leipzig: Teubner; 1978

5 Michaelis E. Albrecht von Graefe. Sein Leben und Wirken. Berlin: G. Reimer; 1877

6 Schilp T, Rohrbach JM. Albrecht von Graefe an Frans Cornelis Donders. Briefe 1852 bis 1870. Essen: Klartext; 2013
7 Rohrbach JM. 1863 - Carl Wilhelm von Zehender und das 1 . Heft der Klinischen Monatsblätter für Augenheilkunde. Klin Monatsbl Augenheilkd 2013; 230: 108-111

8 Hirschberg J. Geschichte der Augenheilkunde. Die Reform der Augenheilkunde, 1. Teil. In: Graefe A, Saemisch T, Hrsg. Handbuch der gesamten Augenheilkunde, 2. Auflage. Berlin: Julius Springer; 1918: 210-314

9 von Graefe A. Mittheilungen vermischten Inhalts. Ophthalmoscopischer Befund bei einem Fall von Microphthalmus congenitus mit Colobom. Graefes Arch Clin Exp Ophthalmol 1855; 2: 239-250 (Die Hinweise auf die glaukomatöse Exkavation finden sich auf den Seiten 248-249)

10 von Graefe A. Ueber Embolie der Arteria centralis retinae als Ursache plötzlicher Erblindung. Graefes Arch Clin Exp Ophthalmol 1859; 5: 136-157

11 von Graefe A. Ueber die Iridectomie bei Glaukom und über den glaucomatösen Process. Graefes Arch Clin Exp Ophthalmol 1857; 3: 456-555

12 Sattler $H$. Klassiker der Medizin. Albrecht von Graefe's grundlegende Arbeiten über den Heilwert der Iridektomie bei Glaukom. Leipzig: Johann Ambrosius Barth; 1911

13 von Graefe A. Beiträge zur Pathologie und Therapie des Glaucoms. Graefes Arch Clin Exp Ophthalmol 1869; 15: 108-252

14 von Graefe A. Vorwort zum 1. Band. Graefes Arch Clin Exp Ophthalmol 1854; 1

15 Rohrbach JM. Ophthalmopathologie zwischen gestern und morgen. Klin Monatsbl Augenheilkd 2004; 221: 87-91

16 Brücke E. Anatomische Beschreibung des menschlichen Augapfels. Berlin: G. Reimer; 1847

17 Quidde M. Erinnerungen an Albrecht von Graefe. Zu seinem 25. Todestage zusammengestellt aus Werken und Briefen J. Jacobson's. Königsberg: Wilhelm Koch; 1895: 87-91

18 Müller $H$. Anatomische Beiträge zur Ophthalmologie. Beschreibung einiger von Prof. v. Graefe exstirpirter Augäpfel. Graefes Arch Clin Exp Ophthalmol 1858; 4: 363-388

19 Müller H. Anatomische Beiträge zur Ophthalmologie. Ueber Niveau-Veränderungen an der Eintrittsstelle des Sehnerven. Graefes Arch Clin Exp Ophthalmol 1858; 4: 1-10

20 Neuhann $W$. Ein unbekannter Zweig im Stammbaum Albrecht von Graefes. In: Hartmann C, Hrsg. Albrecht von Graefe, Berlin 1828 bis 1870 . Germering: ad manum medici; 1996: 103-115

21 Hirschberg J. Geschichte der Augenheilkunde. Die Reform der Augenheilkunde, 2. Teil. In: Graefe A, Saemisch T, Hrsg. Handbuch der gesamten Augenheilkunde, 2. Auflage. Berlin: Julius Springer; 1918: 243-255

22 Müller $H$. Zur Histologie der Netzhaut. Zeitschr Wissenschaftl Zoologie 1851; 3: 234-237

23 Müller $H$. Anatomisch-physiologische Untersuchungen über die Retina des Menschen und der Wirbelthiere. Leipzig: Wilhelm Engelmann; 1856

24 Müller $H$. Anatomische Beiträge zur Ophthalmologie. Ueber einen ringförmigen Muskel am Ciliar-Körper des Menschen und über den Mechanismus der Accommo- dation. Graefes Arch Clin Exp Ophthalmol 1857; 3: 1-24

25 Zehender CW. Brief Albrecht von Graefes vom 24. Mai 1864 an den Herausgeber der „Klinischen Monatsblätter“ (Carl Wilhelm von Zehender), den Tod Heinrich Müllers betreffend. Klin Monatsbl Augenheilkd 1895; 33: 336-337

26 Schweigger C. Pathologisch-anatomische Untersuchungen. Graefes Arch Clin Exp Ophthalmol 1859; 5: 216-240

27 Leber T. Beiträge zur Kenntniss der atrophischen Veränderungen des Sehnerven nebst Bemerkungen über die normale Structur der Nerven. Graefes Arch Clin Exp Ophthalmol 1868; 14: 164-220

28 Hirschberg J. Anatomische Untersuchungen über Glioma retinae. Graefes Arch Clin Exp Ophthalmol 1868; 14: 30-102

29 von Graefe A. Tumor im submucösen Gewebe der Lid-Bindehaut von eigenthümlicher Beschaffenheit. Klin Monatsbl Augenheilkd 1863; $1: 23$

30 von Graefe Av. Zur Casuistik der Tumoren. Graefes Arch Clin Exp Ophthalmol 1864; 10: $176-220$

31 Graefe A. Vorwort. Beiträge zur anatomischen Klinik der Augenkrankheiten. Graefes Arch Clin Exp Ophthalmol 1860; 6: 116-134

32 von Graefe A. Mittheilungen vermischten Inhalts. Präparat von Chorioiditis tuberculosa bei einem Schweine. Graefes Arch Clin Exp Ophthalmol 1855; 2: 210-214

33 Müller $H$. Anatomische Beiträge zur Ophthalmologie. Untersuchungen über die Glashäute des Auges, insbesondere die Glaslamelle der Chorioidea und ihre senilen Veränderungen. Graefes Arch Clin Exp Ophthalmol 1856; 2: 1-65

34 Liebreich $R$. Histologisch-ophthalmoscopische Notizen. Graefes Arch Clin Exp Ophthalmol 1858; 4: 286-303

35 Rohrbach JM, Auw-Hädrich C, Messmer EM et al. Zur Situation der Ophthalmopathologie in Deutschland: eine aktuelle Bestandsaufnahme. Klin Monatsbl Augenheilkd 2009; 226: $740-746$

\section{Bibliografie}

Dol http://dx.doi.org/10.1055/s-0035-1545738 Online-publiziert: 8.4.2015

Klin Monatsbl Augenheilkd 2015; 232: 1101-1104 (c) Georg Thieme Verlag KG Stuttgart · New York . ISSN 0023-2165

\section{Korrespondenzadresse}

Prof. Dr. Jens Martin Rohrbach

Department für Augenheilkunde, Forschungsbereich Geschichte der Augenheilkunde/ Ophthalmopathologisches Labor

Eberhard-Karls-Universität Tübingen

Schleichstraße 6-12

72076 Tübingen

Tel.: + 49/(0)7071/2984761

Fax: + 49/(0)7071/294762

martin.rohrbach@med.uni-tuebingen.de 